\title{
Chemical Proteomics Applied to Target Identification and Drug Discovery
}

\author{
Steven H.L. Verhelst and Matthew Bogyo \\ Stanford University School of Medicine, Stanford, CA, USA
}

\section{Introduction}

In the past decade, DNA sequencing of multiple organisms has provided us with our first look at complete lists of protein products expressed from a whole genome. The field of proteomics is now challenged with the task of elucidating the cellular functions of these proteins in both normal and pathological processes (1-3). Several proteomic techniques, such as 2-dimensional gel-electrophoresis (4) and isotope-coded affinity-tagging (5), provide a means to globally monitor changes in protein expression levels. However, the abundance of a protein is not necessarily directly coupled to its activity. Most enzymes are mediators of biochemical transformations and are therefore highly posttranslationally regulated. Many are synthesized as zymogens, which are tightly regulated through enzymatic activation. Moreover, the activity of enzymes is altered by changes in $\mathrm{pH}$ and the presence of inhibitors. Thus, methods that allow direct quantification of protein activities rather than simple abundance are required to uncover distinct protein functions in physiological and pathological events.

A new research field has been created to begin to address protein function at the level of regulation of enzyme activity. This new area has been given the name chemical proteomics, or activity-based proteomics, and makes use of small molecules that can covalently attach to catalytic residues in an enzyme active site (6-11). The selectivity of the chemically reactive group allows specific proteins or protein subsets to be tagged, purified, and analyzed. As a result, this technique is able to identify novel enzymatic proteins and has the potential to accelerate the discovery of new drug targets.

\section{Activity-Based Probe Design}

The small molecules used in chemical proteomics, so-called activity-based probes (ABPs), generally consist of three basic elements (Figure 1). The warhead consists of a reactive functional group that is able to covalently attach to the active site of an enzyme, while the tag can be used for identification and purification of modified enzymes. The linker connects these two elements, provides selective binding interactions, and prevents steric congestion.

The warhead is perhaps to most critical component of an ABP. On one hand, it should be reactive enough to modify somewhat latent nucleophiles. However, its chemical reactivity must not lead to nonspecific modification of other abundant molecules within the proteome. The majority of ABPs use electrophilic groups, often derived from irreversible enzyme inhibitors (12) that are able to form a covalent bond with a nucleophile located in the enzyme active site. Two examples include the cysteine protease probe DCG-04 (13) and the serine hydrolase probe FP-biotin (14) (Figure 1). The former is derived from the natural product E-64, a broad-spectrum cysteine protease inhibitor. The warhead of the latter resembles the general serine protease inhibitor diisopropyl fluorophosphate.

Besides being a simple connection between warhead and tag, the linker can influence the specificity of the ABP. In this respect, polypeptides are often used as linkers due to their ease of preparation and compatibility with solid phase chemistry. In addition, many enzymes have protein substrates and therefore bind probes with peptide character. Furthermore, information regarding substrate specificity of a given enzyme can be used to design probes that display selectivity for distinct enzyme targets. For instance, the leucine residue in DCG-04, situated next to the epoxide electrophile, directs its selectivity towards the papain family of cysteine proteases, all of which possess hydrophobic P2 pockets. On the other hand, FP-biotin was designed as a general serine hydrolase probe. Therefore, a simple alkyl spacer was chosen to connect the fluorophosphonate to the reporter biotin-group. Consequently, this probe is not limited to a single subclass, but exhibits reactivity towards a broad range of serine hydrolases.

Besides the above described approach using electrophilic warheads, two other strategies have been developed for the design of ABPs. Both of these methods rely on the generation of a highly reactive electrophile in the active site leading to alkylation of a nearby nucleophile on the target. The first class of affinitybased labeling probes contains photolabile groups that can be converted into a highly reactive intermediate after irradiation with UV light $(15,16)$. This class of compounds has been used to label metallo-proteases that do not use a side-chain nucleophile to directly attack a protein substrate. The second class consists of a masked electrophile that becomes activated upon cleavage by the enzyme (17-20). These reagents serve as bona fide enzyme substrates and only become converted into reactive probes after turnover. While this method seems to have promise, it is not yet clear if product release is too rapid, thereby leading to highly nonspecific labeling of other proteins.

Several different tagging strategies can be used to trace proteins that have been targeted by ABPs. In most cases, tags facili-

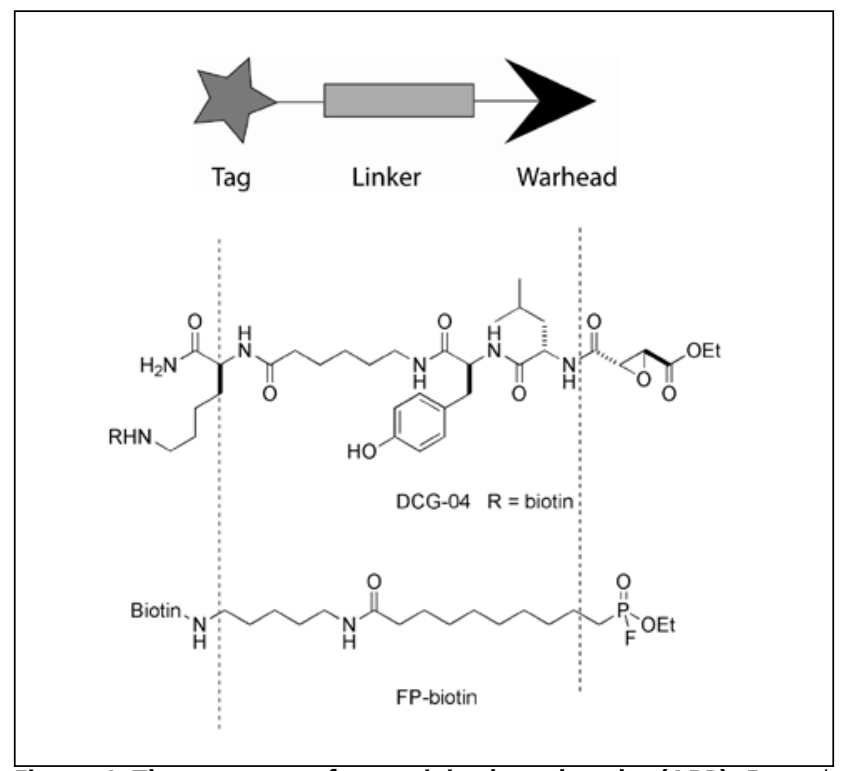

Figure 1. The anatomy of an activity-based probe (ABP). Examples of two probes that use different reactive warheads, different linkers, and the same biotin tag. These probes target cysteine proteases (DCG-04) and serine hydrolases (FP-Biotin) 


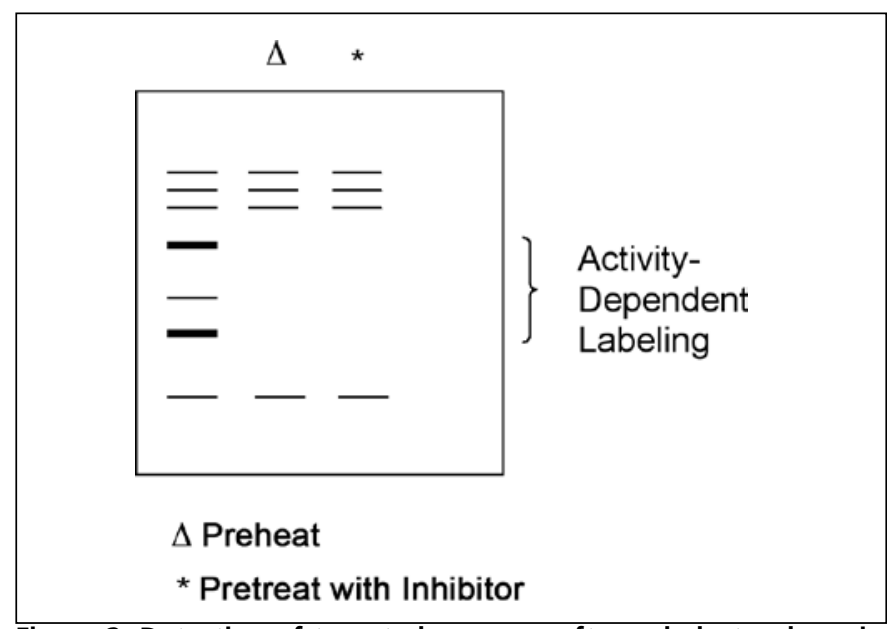

Figure 2. Detection of targeted enzymes after gel electrophoresis. Example of a sodium dodecyl sulfate polyacrylamide gel electrophoresis (SDSPAGE) showing the labeling of a crude protein extract with an activity-based probe (ABP). Lane 1, no label. To determine the ABP-labeled targets resulting from specific enzyme activity, the samples are first preheated $(\Delta)$ or treated with a general inhibitor ${ }^{*}$ ) and then labeled with the ABP. The bands that are lost by pretreatment are the result of specific activity-dependant labeling.

tate the detection of probe-labeled targets after gel electrophoresis. Controls, in which the enzyme activity is abolished, are required to determine the specific labeling patterns. Preheating of samples to denature enzymes or addition of inhibitors are commonly used controls (Figure 2). Radioactive ABPs normally display high sensitivity and low background labeling. Fluorophoric tags are a valuable alternative to radioisotopes as they can be as sensitive as radioactive tags and can be analyzed by direct scanning of gels using laser scanning imaging systems. In addition, these probes can be utilized for in vivo imaging of protease activity, as their presence can be monitored by fluorescent microscopy. Recently, cathepsin activity was monitored in a transgenic mouse model for pancreatic cancer using a fluorescent ABP (21). Biotinylated ABPs facilitate both visualization and purification of targets and have been used for characterization of labeled proteins by in-gel digestion and tandem mass spectrometry analysis.

\section{The Application of ABPs in Target Identification and Drug Discovery}

One of the first steps in the development of new small molecule therapeutics is the selection of a suitable target whose biological activity can be directly linked to a pathological process. Several diseases such as cancer, rheumatoid arthritis, and osteoporosis are associated with elevated levels of protease activity. ABPs can aid in the monitoring and profiling of protease activities throughout disease stages. The resulting information can be used to link a cellular phenotype to up- or down-regulated enzyme activities. Recently, general serine hydrolase ABPs have been used to profile enzyme activities in a diverse range of cancer cell lines $(22,23)$. In studies comparing metastatic and non-metastatic human breast cancer models, it was shown that the former exhibited a higher activity of a $\gamma$-glutathione-Stransferase, an enzyme that has not previously been associated with breast cancer. Furthermore, these general profiling studies suggest that disease phenotypes can be predicted by activity profiling of sets of relevant enzymes. This has great promise for application of ABPs to the identification of novel biomarkers for human disease.

Additionally, ABPs can be used to develop robust screens for small molecule inhibitors of a specific enzyme target within a large family of related enzymes. This method of inhibitor screening allows compounds to be assayed for both potency and selectivity against a set of related targets in complex biological samples. For example, the general papain probe DCG-04 was used to screen inhibitor libraries designed to target cysteine proteases in the human malaria parasite Plasmodium falciparum (24). Compounds in the libraries contained an epoxide warhead linked to natural and non-natural hydrophobic amino acids in a peptide scaffold. These amino acids were varied to generate diverse inhibitors that were screened to identify residues that showed selectivity for individual protease targets. Incubation of parasite lysates with the libraries resulted in inhibition of cysteine proteases by covalent modification of the catalytic cysteine residue. The potency and selectivity of compounds was determined by labeling with the ABP DCG-04, followed by gel electrophoresis analysis. This method identified inhibitors that block either one or multiple proteases. Importantly, multiple hydrophobic nonnatural amino acids were identified and, when placed next to the epoxide scaffold, resulted in selective inhibition of falcipain1 , one of the primary papain-like proteases found in the parasite genome. One of these inhibitors showed greater than 25-fold selectivity for falcipain-1 and led to a block in parasite invasion of host cells when applied to parasite cultures. These findings suggest that falcipain-1 may be an attractive target for therapeutics directed against malaria and highlight the utility of ABPs for functional studies in complex biological systems.

Although the field of chemical proteomics has proven its value in several biologically and pathologically relevant systems, several challenges still remain to be overcome. First, the spectrum of available ABPs needs to be broadened in order to target additional enzyme classes. In addition, the development of highthroughput and nongel-based assays in conjunction with ABPs will be required to enhance the experimental value of chemical proteomics. It is likely that further development of these methods will improve the applications of chemical proteomics to drug discovery research.

\section{Acknowledgments}

The authors thank the Netherlands Organization for Scientific Research (NWO) for financial support (S.V.) and a National Technology Centers for Networks and Pathways grant, National Institutes of Health (NIH) grant no. U54 RR020843 (M.B.).

\section{References}

1. Patterson, S.D. and R.H. Aebersold. 2003. Proteomics: the first decade and beyond. Nat. Genet. 33:R311-R323.

2. Hunter, T.C., N.L. Andon, A. Koller, J.R. Yates III, and P.A. Haynes. 2002. The functional proteomics toolbox: methods and applications. J. Chromatogr. B 782:165-181.

3. Yanagida, M. 2002. Functional proteomics; current achievements. J. Chromatogr. B 771:89-106.

4. Rabilloud, T. 2002. Two-dimensional gel electrophoresis in proteomics: old, old fashioned, but it still climbs up the mountains. Proteomics 2:3-10.

5. Flory, M.R., R.J. Griffin, D. Martin, and R. Aebersold. 2002. Advances in quantitative proteomics using stable isotope tags. Trends Biotechnol. 20:523-529.

6. Jessani, N. and B.F. Cravatt. 2004. The development and application of methods for activity-based protein profiling. Curr. Opin. Chem. Biol. 8:5459.

7. Speers, A.E. and B.F. Cravatt. 2004. Chemical strategies for activitybased proteomics. Chembiochem. 5:41-47. 
8. Jeffery, D.A. and M. Bogyo. 2003. Chemical proteomics and its application to drug discovery. Curr. Opin. Biotechnol. 14:87-95.

9. Campbell, D.A. and A.K. Szardenings. 2003. Functional profiling of the proteome with affinity labels. Curr. Opin. Chem. Biol. 7:296-303.

10.Kozarich, J.W. 2003. Activity-based proteomics: enzyme chemistry redux. Curr. Opin. Chem. Biol. 7:78-83.

11.Huang, X., E.L.P. Tan, G.Y.J. Chen, and S.Q. Yao. 2003. Enzyme-targeting small-molecule probes for proteomics applications. Appl. Gen. Proteomics 2:225-238.

12.Powers, J.C., J.L. Asgian, O.D. Ekici, and K.E. James. 2002. Irreversible inhibitors of serine, cysteine, and threonine proteases. Chem. Rev. 102:4639-4750.

13.Greenbaum, D., K.F. Medzihradszky, A. Burlingame, and M. Bogyo. 2000. Epoxide electrophiles as activity-dependent cysteine protease profiling and discovery tools. Chem. Biol. 7:569-581.

14.Liu, Y., M.P. Patricelli, and B.F. Cravatt. 1999. Activity-based protein profiling: the serine hydrolases. Proc. Natl. Acad. Sci. USA 96:1469414699.

15.Saghatelian, A., N. Jessani, A. Joseph, M. Humphrey, and B.F. Cravatt. 2004. Activity-based probes for the proteomic profiling of metalloproteases. Proc. Natl. Acad. Sci. USA 101:10000-10005.

16.Chan, E.W.S., S. Chattopadhaya, R.C. Panicker, X. Huang, and S.Q. Yao. 2004. Developing photoactive affinity probes for proteomic profiling: hydroxamate-based probes for metalloproteases. J. Am. Chem. Soc. 126:14435-14446.

17.Zhu, Q., A. Girish, S. Chattopadhaya, and S.Q. Yao. 2004. Developing novel activity-based fluorescent probes that target different classes of proteases. Chem. Commun. 7:1512-1513.

18.Lo, L.-C., R.-L. Pang, C.-H. Kuo, Y.-L. Chiang, H.-Y. Wang, and J.-J. Lin. 2002. Design and synthesis of class-selective activity probes for protein tyrosine phosphatases. J. Proteome Res. 1:35-40.

19.Zhu, Q., X. Huang, G.Y.J. Chen, and S.Q. Yao. 2003. Activity-based fluorescent probes that target phosphatases. Tetrahedron Lett. 44:26692672.

20.Tsai, C.-S., Y.-K. Li, and L.-C. Lo. 2002. Design and synthesis of activity probes for glycosidases. Org. Lett. 4:3607-3610.

21.Joyce, J.A., A. Baruch, K. Chehade, N. Meyer-Morse, F. Giraudo, F.Y. Tsai, D. C. Greenbaum, J. H. Hager, et al. 2004. Cathepsin cysteine proteases are effectors of invasive growth and angiogenesis during multistage tumorigenesis. Cancer Cell 5:443-453.

22.Adam, G.C., B.F. Cravatt, and E.J. Sorensen. 2001. Profiling the specific reactivity of the proteome with non-directed activity-based probes. Chem. Biol. 8:81-95.

23.Adam, G.C., E.J. Sorensen, and B.F. Cravatt. 2002. Proteomic profiling of mechanistically distinct enzyme classes using a common chemotype. Nat. Biotechnol. 20:805-809.

24.Greenbaum, D.C., A. Baruch, M. Grainger, Z. Bozdech, K.F. Medzihradszky, J. Engel, J. DeRisi, A.A. Holder, and M. Bogyo. 2002. A role for the protease falcipain 1 in host cell invasion by the human malaria parasite. Science 298:2002-2006. 